\title{
Voxel-Based Sensor Placement for Additive Manufacturing Applications
}

\author{
Nuwan Munasinghe - Thomas Romeijn - Gavin Paul
}

Received: date / Accepted: date

\begin{abstract}
The manufacturing sector is experiencing a transformation to Industry 4.0. The authors are researching 3D printable sensors to measure operating conditions like wear, strain and temperature, as part of a project to develop a large-scale multi-material extrusion 3D printer to print a Gravity Separation Spiral (GSS) - a piece of mining equipment that separates minerals from slurry. This paper proposes a sensor placement methodology for placing embedded sensors in large 3D printed objects. Voxels, the 3D equivalent of $2 \mathrm{D}$ pixels, are used to discretise the object and an optimisation routine optimally positions the $3 \mathrm{D}$ printable sensors into a 3D printed object. The optimisation objectives that are used during sensor placement include the information gain from the sensor, the ability to print the sensor using a robot, and the ability to discourage sensors being placed in important structural locations by penalising these voxels. Finite element analysis is employed to measure the information gain, while the robot arm's manipulability measures the capability to print at each voxel location. The objectives are integrated using 3D kernels, which are represented by voxels shaped in the size of the sensor and different weights related to the intricate traces that need to be printed. Using a weighted objective function the best locations are chosen. A simulation environment has been devel-
\end{abstract}

This research is supported by UTS, in particular, Rapido; The Commonwealth of Australia's Department of Industry, Innovation and Science (Innovative Manufacturing CRC Ltd); and Downer, via its subsidiary Mineral Technologies.

$\triangle$ Nuwan Munasinghe ${ }^{1}$, Thomas Romeijn ${ }^{2}$, Gavin Paul ${ }^{3}$

Centre for Autonomous Systems

University of Technology Sydney

Australia

E-mail: $\quad\left\{{ }^{1}\right.$ Nuwan.Munasinghe,${ }^{3}$ Gavin.Paul $\} @ u t s . e d u . a u$,

${ }^{2}$ Thomas.Romeijn@student.uts.edu.au oped to simulate the printing and Matlab is used to do the voxel-based calculations to identify the ideal location for sensor placement.

Keywords Advanced manufacturing · Additive manufacturing $\cdot 3 \mathrm{D}$ printing $\cdot$ Sensor placement

\section{Introduction}

The fourth industrial revolution, Industry 4.0 is providing businesses with the opportunity to create new products and services to expand supply chains [1]. With the introduction of the Internet of Things (IoT) and cyber-physical systems (CPSs) in the industrial domain, industrial automation is undergoing a tremendous change.

Additive manufacturing (AM), widely known as $3 \mathrm{D}$ printing, is a method of creating a physical object from a 3D model by depositing material layerby-layer [2,3]. In recent years, AM has evolved from a rapid prototyping tool to an end-product manufacturing method 4. To overcome inherent drawbacks in traditional mould-based manufacturing, a research and development project is underway to develop a multimaterial extrusion 2] 3D printer with two industrial robot arms to manufacture Gravity Separation Spirals (GSS). These GSS are used in the mining industry to separate minerals from a slurry. When a slurry is poured into the spiral from the top, gravity and the shape of the spiral separate minerals according to their specific weight. The profile of the spiral is designed to target the intended mineral type. GSS are traditionally manufactured using mould-based techniques using material like fibreglass and polyurethane. Customising the shape of the spiral is uneconomical and time-consuming because of the need to create new moulds and the manual labour 


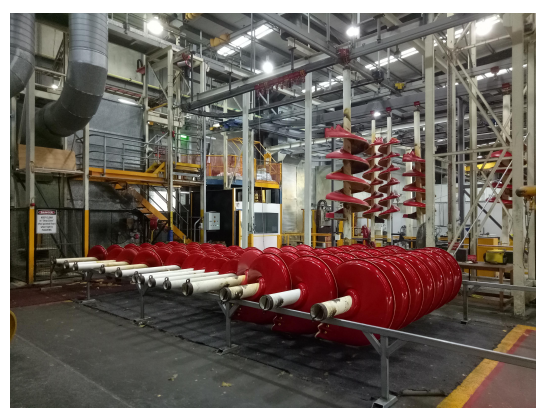

(a)

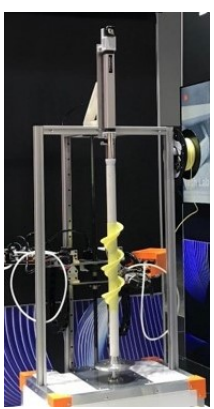

(b)

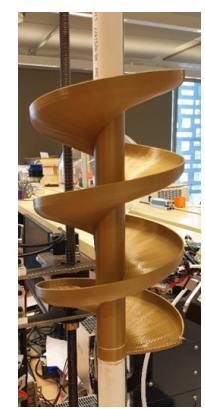

(c)

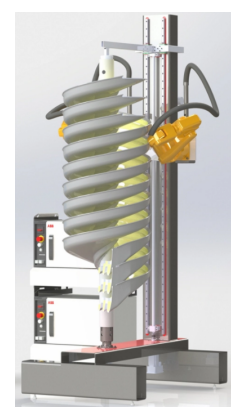

(d)

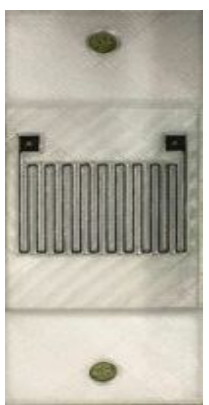

(e)

Fig. 1: a) GSS manufacturing factory. b) Small scale (1:3) printer. c) Printed spiral using small scale printer. d) Full-scale printer. e) 3D printed strain gauge using polylactic acid (PLA) base and carbon-based PLA for conductive traces with extrusion printing.

required. Since current manufacturing methods require manual labour, there is a potential health risk of exposing workers to hazardous materials. Fig. 1 1 a shows a GSS manufacturing factory. A large-scale multi-material extrusion printer is under development, which can be used to print different polymer material like Polyamide (PA), Thermoplastic polyurethane (TPU) and Polyethylene terephthalate glycol-modified (PETG). There is a separate research project underway to identify the best material based on abrasion, creep and accelerated weathering tests. A team in UTS Rapido in collaboration with Downer's Mineral Technologies, supported by the Australian Innovative Manufacturing CRC, have developed a 1:3 scale 3D printer as shown in Fig. 1 1 . A printed spiral using this printer is shown in Fig. 11., and current development towards the full-scale printer is shown in Fig. 1 $1 \mathrm{~d}$. The full-scale printer has a $3 \mathrm{~mm}$ nozzle diameter, print width of $5 \mathrm{~mm}$ and print accuracy of $\pm 0.3 \mathrm{~mm}$. Since the printing process is executed radially in vertical layers without any support structures, a novel radial slicing algorithm and a tool path planning algorithm have been developed for this specific application 6, 7].

These GSS operate in various remote parts of countries. To make the GSS equipment more capable and "smart", different sensors are under development that can be printed using extrusion-based printing inline or mounted externally onto the spiral to measure various parameters like temperature [8, wear [9] and strain [10, 11. Fig. 1e shows a 3D printed strain gauge printed using an extrusion-based method and using carbon-based conductive PLA and PLA base. Monitoring the operating conditions remotely allows for remote fault diagnosis, which reduces the need to send technicians to these remote locations, and enables operational efficiency improvements through a real-time and detailed history of usage. Placing conventional sensors (e.g. strain) inside printed GSS requires either pausing the print and placing them, making poor layer adhesion, or making a cavity to place them after the print, which changes the surface quality of the GSS, thus reducing the performance of mineral separation. Depending on the location, making such a cavity might cause structural integrity issues in the printed structure. For all 3D printed objects, printing sensors might not be ideal depending on the print method, material and the nature of the object. This paper focuses on providing a methodology for sensor placement in 3D printed objects where printing sensors have been chosen based on the print method, material, sensor performance and the printer kinematics.

Structural health monitoring (SHM) can be defined as the process of implementing methods to detect damages of various structures in civil, aerospace and mechanical engineering [12,13. SHM plays a significant role in detecting damages in large and complex structures to ensure serviceability and sustainability [12]. SHM systems help evaluate the conditions of the structure in order to avoid failures and to help to plan maintenance actions without operation disruptions [14. GSS operate constantly in mining sites and identifying problems early will help to maintain continuous operation. Optimal sensor placement (OSP) directly impacts the quality of the collected data and is, therefore, a critical aspect in SHM 12. Without OSP, there is a risk of false-positive detections that could result in unnecessary closure for maintenance, and affect operational costs [14. OSP helps to reduce the cost of the SHM systems without compromising the quality of the monitoring approach [14]. Additionally, OSP provides a minimum number of sensor locations that will provide adequate information to identify the required data [15].

In this research, finite element analysis (FEA) has been used to obtain a distribution of the parameter of 
interest in the structure. Robert et al. [16] developed a method for an optimal layout design of sensor arrays for SHM under uncertainty which includes FEA. Robert et al. incorporated FEA, structural damage detection algorithms and reliability-based optimisation concepts. FEA-based OSP has been used to place sensors in buildings. Ting et al. 17] used FEA-based sensor placement for the Dalian World Trade Building, and since the number of degrees-of-freedom (DOF) is high, they used a simplified multi-DOF system. Kammer [18] addressed the sensor placement problem in large space structures (LSS) from the standpoint of structural dynamics and used collected data from the sensors to validate the LSS finite element model (FEM). The updated FEM more accurately describes the real structure.

The concept of the information gained from a sensor in a certain location is a promising way to approach sensor placement. Costas et al. 19] used information entropy (IE) as a performance measure of the sensor configuration. In their method, optimal sensor configuration is designed as an optimisation problem, including discrete-valued variables, which is then solved using sequential sensor placement algorithms. By selecting the sensor setting which minimises the IE, it becomes possible to select an optimal sensor configuration [20]. Meo et al. 21] used a method to maximise the information by employing OSP methods based on covariance matrix coefficients and energetic approaches.

There are various types of algorithms developed for sensor placement. General sensor selection problems addressing reliability, observability, detectability and diagnosability are NP-complete and therefore, computationally expensive. Thus, other researchers have used constraint-based objective function optimisation methods to reduce the computation complexity [15]. Additionally, different methods have been developed to reduce the complexity of complicated optimisation methods [22]. However, conventional gradient-based optimisation methods are unable to handle multiple local optimums efficiently and have difficulty in estimating global minimum, and in recent years, OSP biological optimisation techniques have been applied [15]. Other similar methods used for OSP are particle swarm optimisation and ant colony optimisation 23,24. Sequential sensor placement method is another algorithm which is a systematic and computationally efficient approach for obtaining better sensor configurations [14]. However, it cannot be guaranteed to be the most optimum method 25. In this method, a fixed number of sensors are placed in a given number of positions and computed sequentially by placing one sensor at a time that results in the highest reduction in the objective function [15. The proposed sensor placement method in this paper is an outranking multi-criterion decision-making method similar to Preference Ranking Organisation Method for Enrichment Evaluations (PROMETHEE) and Elimination and Choice Expressing Reality (ELECTRE). PROMETHEE provides a ranking of a finite number of alternatives from best to worst [26 and ELECTRE lists potential alternatives and evaluates these using criteria defined by the user 27. Unlike classic optimisation methods, where a problem is formulated to a cost function for an optimum, this method compares multiple solutions criterion by criterion [27. In the problem addressed in this paper, there is a finite number of sensor locations that need to be evaluated according to the criteria defined in the objective function similar to these methods.

In the area of sensor placement in 3D printed objects, Hwang at al. 28] used tactile objects covered with black ink to experimentally identify areas where sensors should be placed in $3 \mathrm{D}$ printed prosthetic hands. In this method, after touching objects covered with ink, areas on the gloved hand that had ink stains were identified using image processing and recommended as ideal areas for sensor placement on the prosthetic hands.

In this paper, a weighted approach was proposed to combine multiple criteria for OSP. Values of the weights and the computation of those weights play a fundamental role and they depend on the importance of each criterion, which in turn depends on the application. This paper does not suggest a specific approach to calculate these weights. However, there are few methods to do this. One method is similar to the method used in the Analytic Hierarchy Process (AHP) which uses a pairwise comparison matrix to compare different criteria and calculate weights 29. In the ELECTRE method, where alternatives are evaluated using criteria, the user provides a weight to each one increasing with its importance [27. In the PROMETHEE method, determination of weights is an important step and assumes that the decision-maker able to weigh the criteria properly [26. The method proposed in this paper to aggregate different criteria is quite similar to PROMETHEE and, different to AHP and ELECTRE method as it considers pair-wise comparisons to compare different criterion. However, it is possible to use a systematic weight calculation method like in AHP to calculate the weights.

A sensor placement method that is suitable for a particular application is not necessarily suitable for other applications [15. Decisions about where a 3D printed embedded sensor should be located within a larger 3D printed object is novel and different from conventional sensor placement problems. There are additional factors that need to be considered in this application. One factor is the ability of the robot arm to print 


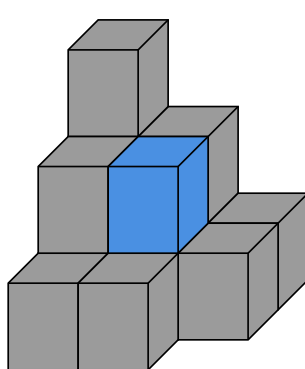

(a)

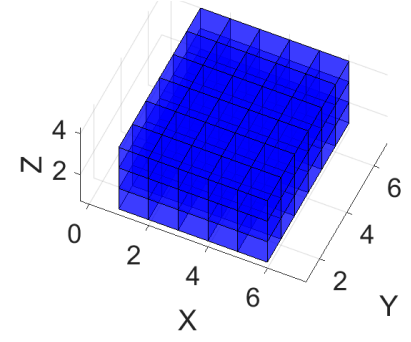

(b)

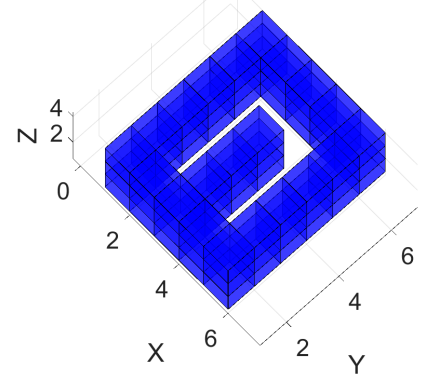

(c)

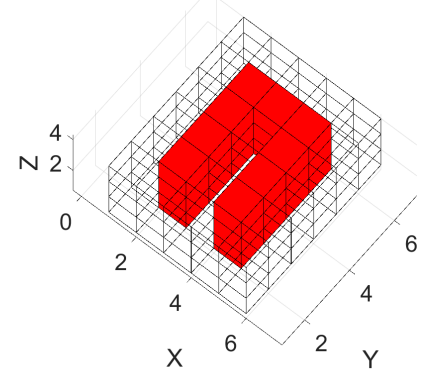

(d)

Fig. 2: a) Set of voxels with one voxel shaded. b) 3D voxels in a size of $3 \times 6 \times 5$. c) Base voxels. d) Sensor voxels.

a sensor in a given place. The ability to print in a given $3 \mathrm{D}$ location can be determined by a robots manipulability measure and this idea of "printability" has been investigated for the project's 3D printer in prior work [6. The printability measure has been used to quantify the ability to print the 3D printed sensors in a particular place. Another factor is the design of the sensor. Since traces (refer to dark lines in Fig. 11) in sensors needs to printed inline, the way they are arranged is important. Different sensor designs printing at the same location require different dexterity levels for the robot arm since the print head follows different paths. Therefore, the sensor design should be considered as a factor in the sensor placement problem. A further factor is the need to forbid or discourage sensor placement in certain areas based on various reasons such as the structural impact from the sensor to the overall structure. Since printing a sensor with a different type of material in a load-bearing structure can reduce its structural integrity, this aspect needs to be considered as well.

This paper presents a theoretical methodology about a novel 3D printed sensor placement algorithm for 3D printed objects using voxels where printing sensors is ideal and chosen for the application. Voxels can be described as the $3 \mathrm{D}$ equivalent of $2 \mathrm{D}$ pixels [30. $2 \mathrm{D}$ kernels have been used in image processing [31 and, this paper proposes a way for voxels to be considered as an extension of $2 \mathrm{D}$ kernels to $3 \mathrm{D}$ space for sensor placement. The specific contributions is a sensor placement algorithm which considers:

- Shape, volume and the design of the sensor.

- Information gain from the sensor.

- Ability of printing sensors in a given location.

- Discourage sensor placement in certain areas.

The remainder of the paper is organised as follows. Firstly, Section 2 provides a mathematical background and theory related to the work. Section 3 provides results of the experiment, Section 4 provides a discussion about results and finally, Section 5 concludes the paper.

\section{Methodology}

\subsection{Voxels}

Voxels (volume pixels) are a 3D equivalent of 2D pixels 30. They represent a value in a regular grid in 3Dspace. Voxels have been used in various applications like computer graphics, medicine and robot perception 32, 33, 34. The advantage of using voxels is that it reduces computationally expensive steps such as meshing, mapping, volume integration and matrix-based solution methods [35]. Fig. 2a shows set of voxels with one voxel shaded.

\subsection{Extension of 2D Kernels in to 3D Space}

Kernel, in image processing, is a $2 \mathrm{D}$ matrix or a mask used for different operations like blurring, sharpening and edge detection [36. Such a matrix is shown in Fig. 33 , with the weight of a cell, $W_{i, j} \in \mathbb{R}$, row number, $j$ and column number $i$. The idea proposed in this paper is to extend this 2D kernel into 3D space where weight in a voxel, $W_{i, j, k}$ and z-index is $k$. When the 2D kernel in Fig. 3a is extended into 3D space by layering it three times, a 3D kernel with voxels is created as shown in Fig. 2b.

2.3 Incorporating Aspects of Sensor Placement for 3D Printing Using Voxels

The basic idea in this proposed method is to voxelise (convert the 3D object to voxels) the sensor and the object the sensor is to be placed in. This is followed by finding a set of voxels in the shape and size of the sensor and placing them in the object voxel set while considering various constraints. Constraints are represented as a single objective function including all the 
necessary factors to evaluate a given location for sensor placement suitability.

A sensor can be designed to have any arbitrary 3D shape. As an example, consider a strain sensor which has an overall shape of a cuboid with printed conductive traces oriented in a "U" shape. Within this cuboid, two types of voxels have been defined. Voxels that surround the conductive traces are called base voxels (Fig. 2f), and voxels that overlap with conductive traces are called sensor voxels (Fig. 2 $\mathrm{d}$ ). Two figures (Fig. 2r and d) have been used to show these voxels separately but they reside in the same cuboid. The reason for defining two types of voxels is to assign separate values of weights for each; the weight of a base voxel, $W_{B}$ and the weight of a sensor voxel, $W_{S}$. The size of the voxels can be selected depending on the minimum conductive trace width and required granularity. In 3D space, consider a particular distribution of a property, $X_{i, j, k} \in \mathbb{R}$. This property can be one that the sensor is designed to measure, such as strain in this case. The objective of this research is to incorporate aspects of the design of the sensor, such as information gain, printability and the ability to discourage sensor placement in certain areas, into the sensor placement problem. The following sections explain how these different aspects are incorporated using voxels. For clarity, a single-layer voxel set is used as an example. Fig. 3b shows the selected kernel with weight values, where $W_{B}=1$ and $W_{S}=2$. The sensor voxels are highlighted in grey colour. Fig. 3r and d shows an example strain distribution $\left(X_{i, j}\right)$ in two different possible sensor locations.

\subsubsection{Design of the Sensor}

The 3D printed sensor has a shape, volume and an arrangement of conductive traces associated with it. Since conductive traces measure the parameter (e.g. temperature, strain) encompassed by its volume, its size and shape should be considered. Additionally, the volume of the sensor should be considered since the sensor should be able to be placed at a given location within the physical dimensions of the object. To consider this factor, the overall volume and shape of the voxel set is chosen to match the shape and volume of the sensor.

\subsubsection{Information Gain}

The parameter of interest that is to be measured can be distributed in the object in different ways. There can be places in the object at which the parameter values are high enough to be measured. Placing sensors without considering information gain will provide less effective measurements. To estimate the amount of information that can be extracted from the placed sensor, the Finite Element Method (FEM) has been utilised in this research to determine the distribution of the parameter that needs be measured within the body of interest. As conductive traces measure the parameter in the object, a high-value parameter distribution closer to the traces will provide a more accurate measurement. The proposed weighting method accommodates for this. Consider the $2 \mathrm{D}$ kernel $\left(W_{i, j}\right)$ in Fig. $3 \mathrm{p}$ and the two different, 2D distributions of a parameter of interest in Fig. 35 and d. The kernel values in Fig. 3b are denotated as $A_{i, j}$, and the kernel values in Fig. $3 \mathrm{~d}$ as $B_{i, j}$. In both locations, there are an equal numbers of $1 \mathrm{~s}$ and $3 \mathrm{~s}$, hence, $\Sigma A_{i, j}$ and $\Sigma B_{i, j}$ are equal. However, $\Sigma\left(B_{i, j} \cdot W_{i, j}\right)$ has a large value compared to $\Sigma\left(A_{i, j} \cdot W_{i, j}\right)$. Therefore, the proposed weighting kernel method favours location B, even though both locations have the same number of values.

\subsubsection{Printability}

When printing the required sensors inline, it is necessary to print internal patterns with conductive traces that vary depending on the sensor type. Hence, although the selected place may be suitable in terms of the information gain factor, it might be difficult to print the sensor in that specific location. In the bespoke GSS printer, robot manipulators are used to move the hotend nozzles. To quantify the ability to print at a given location, a manipulability measure has been used previously [6. The ability to move a robot manipulator easily (dexterity) in any arbitrary direction in 3D space is referred to as its manipulability 37. The manipulability is a scalar measure where a higher value is better and indicates a higher level of dexterity. This measure is based on the Jacobian matrix of the manipulator. The Jacobian maps joint angular velocity, $\dot{q}$, to the end-effector Cartesian velocity, $v$, as shown in (1), where $q$ is the joint angles [37. This measure is initially proposed by Yoshikawa [38] and is shown in (2). The manipulability value is considered as another distribution in the $3 \mathrm{D}$ space and used with the kernel to evaluate a given voxel location. If the manipulability distribution in a voxel set is $M_{i, j}$, the ability to print at that location (i.e. the printability) can be calculated using $\Sigma\left(M_{i, j} \cdot W_{i, j}\right)$ as before.

$$
\begin{aligned}
& \dot{q}=J(q)^{-1} v \\
& m=\sqrt{\operatorname{det}\left(J J^{T}\right)}
\end{aligned}
$$




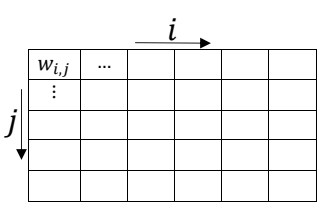

(a)

\begin{tabular}{|l|l|l|l|l|l|}
\hline 1 & 1 & 1 & 1 & 1 & 1 \\
\hline 1 & 2 & 2 & 2 & 2 & 1 \\
\hline 1 & 1 & 1 & 1 & 2 & 1 \\
\hline 1 & 2 & 2 & 2 & 2 & 1 \\
\hline 1 & 1 & 1 & 1 & 1 & 1 \\
\hline
\end{tabular}

(b)

\begin{tabular}{|l|l|l|l|l|l|}
\hline 1 & 3 & 3 & 3 & 3 & 1 \\
\hline 1 & 1 & 1 & 1 & 1 & 1 \\
\hline 1 & 1 & 1 & 1 & 1 & 3 \\
\hline 1 & 1 & 1 & 1 & 1 & 1 \\
\hline 1 & 3 & 3 & 3 & 3 & 1 \\
\hline
\end{tabular}

(c)

\begin{tabular}{|l|l|l|l|l|l|}
\hline 1 & 1 & 1 & 1 & 1 & 1 \\
\hline 1 & 3 & 3 & 3 & 3 & 1 \\
\hline 1 & 1 & 1 & 1 & 3 & 1 \\
\hline 1 & 3 & 3 & 3 & 3 & 1 \\
\hline 1 & 1 & 1 & 1 & 1 & 1 \\
\hline
\end{tabular}

(d)

Fig. 3: a) General 2D kernel. b) Selected kernel. c) Location - A. d) Location - B.

\subsubsection{Penalties to Discourage Sensor Placement}

3D printed sensors might contain different materials to the base material of the structure like carbon-based conductive traces used in developed strain [10,11, wear [9], temperature 8] sensors. Therefore, the sensor might have different mechanical properties than those of the structure depending on the type of the material and printing processes used. Previously conducted experiments in strain sensor printing using the extrusionbased method with PLA base material and carbonbased PLA for conductive material showed different mechanical properties than pure PLA material [11. Therefore depending on the material and printing process, placing a sensor in certain locations might adversely affect the structural characteristics of the larger printed object. This would be problematic in a large, loadbearing structure like a GSS and is thus a requirement to be considered in the sensor placement process. Additionally, there may be places that the sensors should not be placed based on other factors, such as attachment points or for aesthetic reasons. Therefore, if required and depending on the application, areas where sensors cannot be placed should be considered during the sensor placement. To represent these areas, another distribution, $R_{i, j} \in\{0,1\}$ is added to the $3 \mathrm{D}$ kernel, where a 0 value allows the placement of a sensor in the grid location and a 1 value forbids placement. The percentage of penalised voxels in a selected location is calculated as per Eq. (3). The number of voxels in a given location where sensors should not be placed is $n_{r}$, the number of base voxels is $n_{b}$, and the number of sensor voxels is $n_{s}$.

$n_{r} /\left(n_{b}+n_{s}\right)$

\subsection{Steps of Sensor Placement}

The following are the steps in sensor placement incorporating the factors mentioned above and a flow chart of these steps and output of each steps are shown in Fig. 4 .

\subsubsection{Voxelise the 3D Printable Object}

The volume of the printed object is converted into a set of voxels, $V_{o b j}$. The size of the voxel is user-defined and depends on the actual size of the object and the size of the sensor (length $69 \mathrm{~mm}$, width $56 \mathrm{~mm}$, height $7 \mathrm{~mm}$ ).

\subsubsection{Calculate Printability and Information Value Distributions}

Using printing simulation, a point cloud of manipulability values, $m_{x, y, z}$ is generated where $x, y, z$ are the cartesian coordinates. This point cloud contains a Cartesian location and a value of manipulability. The point cloud values are then averaged to each volume in a voxel. For each voxel in the set, there is a lower and upper bound for $x, y, z$ values which are represented by $x^{-}, x^{+}, y^{-}, y^{+}, z^{-}, z^{+}$where $x^{-}<x^{+}, y^{-}<y^{+}, z^{-}<$ $z^{+}$. Then, for each voxel the manipulability values are averaged as shown in Eq. (4), subject to lower and upper bounds, where $n_{m v}$ is the number of manipulability points within the voxel. The normalised manipulability set, $X_{m}$ is a collection of these averaged manipulability values divided by the maximum voxel manipulability value, $m_{\max }$ as shown in Eq. (5), and Eq. (6) where the number of voxels are $n$. Similarly, a point cloud of parameters like strain or temperature can be generated using the results of a FEA so a distribution of information, $X_{I}$ can be calculated.

$m_{v 1}=\frac{\sum m_{x, y, z}}{n_{m v}}$
$m_{\max }=\max \left(m_{v 1}, m_{v 2}, \ldots, m_{v n}\right)$
$X_{m}=\left\{m_{v 1} / m_{\max }, \ldots, m_{v n} / m_{\max }\right\}$

\subsubsection{Select Sensor Location and Sensor Voxel Sets}

From $V_{o b j}$, select the subset of sensor location voxels, $V_{l o c}\left(V_{l o c} \subseteq V_{o b j}\right)$ where each voxel set encapsulates the entire sensor shape and thus necessarily has dimensions which are at least as large as the sensor's dimensions. These voxel sets are spread through the whole object in different orientations. This paper does not propose a 


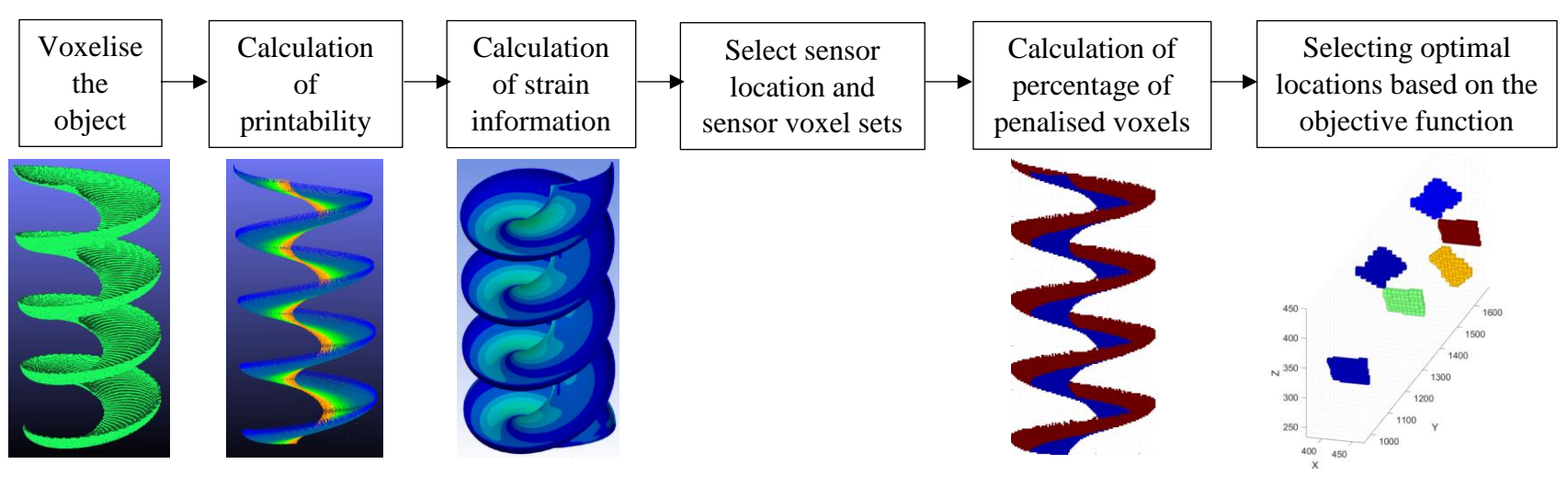

Fig. 4: Flow chart of the process.

method of initial sensor location or arrangement selection. These locations and orientations are guided by the user who is informed by the requirements of the practical application. The user is responsible of selecting suitable initial locations considering things like overlapping sensors. This method simplifies the computations, which is important when optimising for such large-scale objects. These are all candidate sensor locations, where the best ones are selected later. From each voxel location set, $V_{l}\left(V_{l} \in V_{l o c}\right)$, a sensor voxel set, $V_{s}\left(V_{l} \subseteq V_{l o c}\right)$ is selected in the shape of the conductive traces, and the remaining base voxel set is $V_{b}\left(V_{b}=V_{l}-V_{s}\right)$.

\subsubsection{Calculating the Percentage of Penalised Voxels}

From $V_{o b j}$, a set of voxels is selected which is in the areas where sensor placement is forbidden or penalised $V_{r}$. For each $V_{l}$, the percentage of penalised voxels are calculated as in Eq. (7).

\subsubsection{Calculating and Optimising the Objective Function Value for Each Location}

The final step is to incorporate all these factors and evaluate each possible location to select the best locations. To do this, an objective function to evaluate each location has been proposed. When converting a sensor shape to voxels, different locations might have a slightly different number of voxels, since it is an approximation of the original shape. Thus, for a given location, the information and manipulability values have been calculated for the base and sensor voxel sets on a per voxel basis, as shown in Eq. (8) to Eq. (11). Equation 12 shows the objective function where $W_{R}$ is the weight for the penalised voxels. All the weights for different factors can be tuned by the user depending on the importance of the factors.

$$
\begin{aligned}
R_{l} & =\frac{\left|V_{l} \cap V_{r}\right|}{\left|V_{l}\right|} \\
I_{B} & =\frac{\sum X_{I} \cap V_{b}}{\left|V_{b}\right|} \\
I_{S} & =\frac{\sum X_{I} \cap V_{s}}{\left|V_{s}\right|} \\
M_{B} & =\frac{\sum X_{m} \cap V_{b}}{\left|V_{b}\right|} \\
M_{S} & =\frac{\sum X_{m} \cap V_{s}}{\left|V_{s}\right|} \\
C_{l} & =W_{B} \cdot I_{B}+W_{B} \cdot M_{B}+W_{S} \cdot I_{S} \\
& +W_{S} \cdot M_{S}-W_{R} \cdot R_{l}
\end{aligned}
$$

After calculating the objective value for each location voxel sets, the voxel sets are ranked from highest $C_{l}$ value to the lowest. Therefore, the location with highest $C_{l}$ value is the most suitable location according to the criteria and, depending on the requirement, multiple sensor locations can be selected as well from the remaining ranked list.

\section{Results}

This section explains how the presented steps are implemented along with the outputs. ANSYS software has been used to perform the FEA, while Matlab software does both the voxel computations and the robotic simulations with support from the Robotics Toolbox 39. developed by Peter Corke.

\subsection{Voxelisation of the 3D Printable Object}

In this application, the 3D printable object is the GSS. Using a CAD program (SolidWorks), a 3D model of 


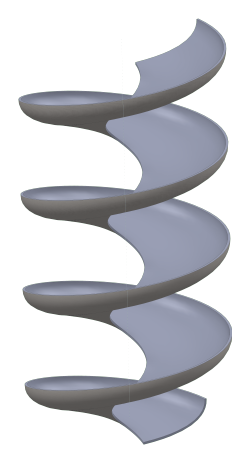

(a)

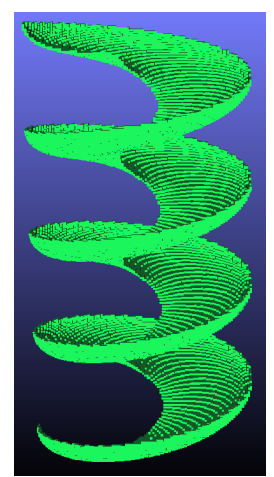

(b)

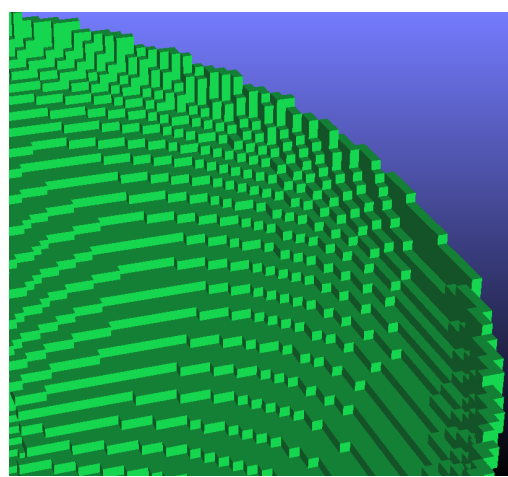

(c)

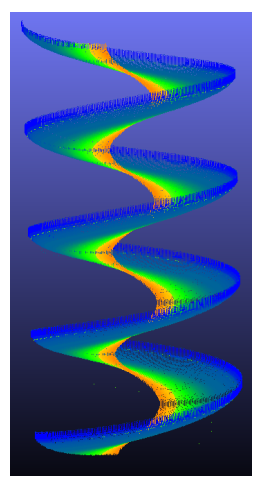

(d)

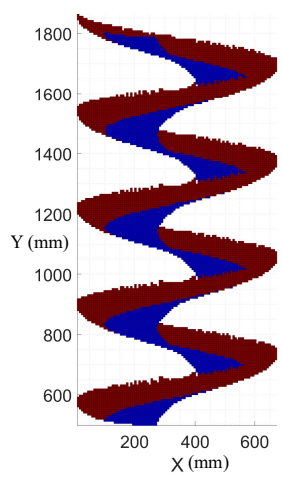

(e)

Fig. 5: a) 3D model of the spiral. b) Voxelised 3D model of the spiral. c) A closer view of the voxels. d) Manipulability point cloud with the colours representing the value of manipulability from orange to green and to blue indicating the transition from high to low manipulability values. e) Example of penalised voxels where sensors placement is discouraged are shown in red.

the GSS is exported to a stereolithography or Standard Triangulation Language (STL) file. This 3D model is shown in Fig. 5 a. The dimensions of the spiral are $0.7 \mathrm{~m}$ x $0.7 \mathrm{~m} \mathrm{x} 1.4 \mathrm{~m}$ for width, length, and height, respectively. The total spiral was voxelised with Matlab into around $100 \times 100 \times 200$ voxels. The voxelised full spiral is shown in Fig. 5b and a closer view of the voxels are shown in Fig. 5 .

\subsection{Calculation of Printability}

A simulation environment has been developed to simulate the robot performing $3 \mathrm{D}$ printing. This environment was developed in Matlab according to the ac- tual dimensions of the printer. The kinematic model of the industrial robots (ABB IRB 120) was defined using Denavit-Hartenberg (DH) parameters and simulated with Peter Corke's Robotics Toolbox. Fig.6] shows the simulated printing of a single trajectory that has been planned using a published radial slicing algorithm 6]. The manipulability values can then be calculated along the print trajectory using Eq. (2). These values are stored with the corresponding cartesian location, and then exported as a rich point cloud, as shown in Fig. 5d. The point cloud is subsequently voxelised (i.e. the printability values are averaged and normalised into voxels), as explained in Section 2.4.2.

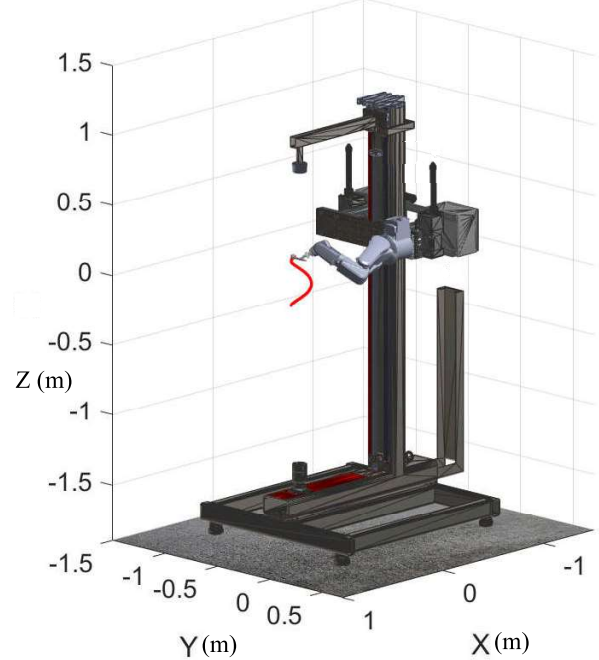

Fig. 6: The simulation used to compute the manipulability information during GSS printing [6].

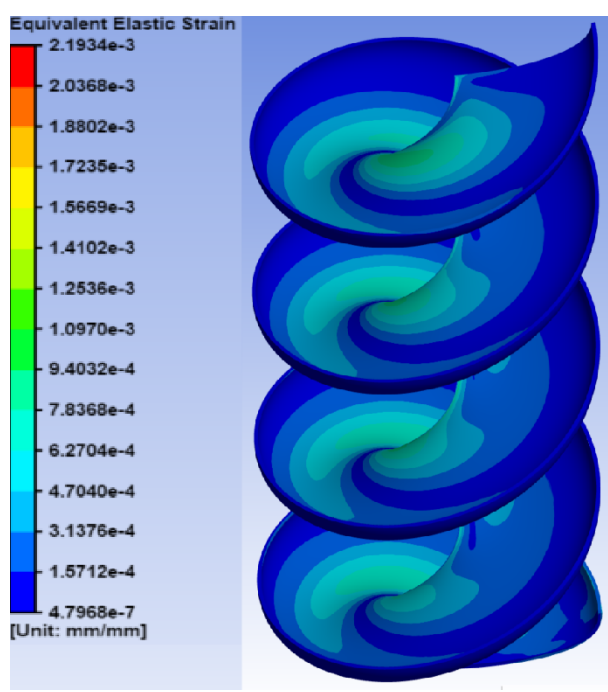

Fig. 7: ANSYS strain simulation result. 


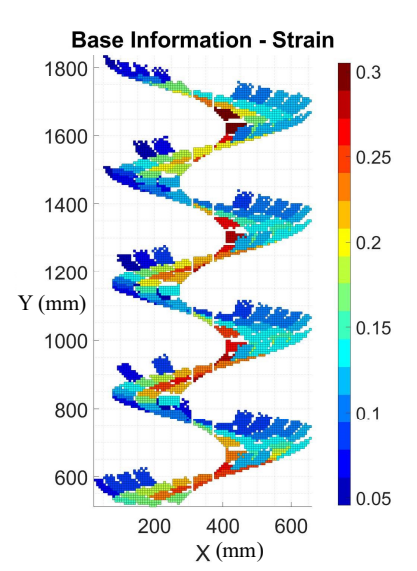

(a)

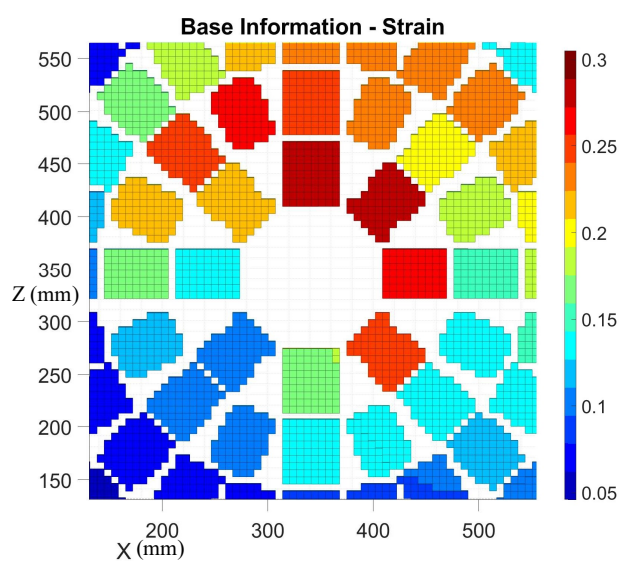

(b)

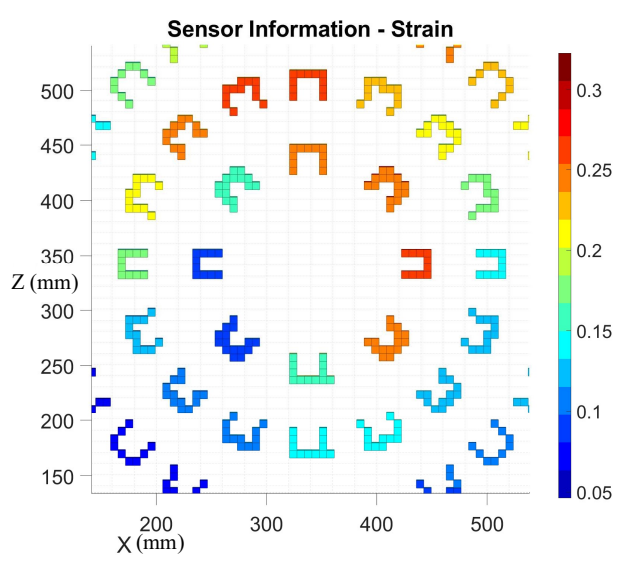

(c)

Fig. 8: Normalised strain values: a) for $V_{l}$; b) for $V_{l}$ in bottom-up view; c) for $V_{s}$ in bottom-up view.

\subsection{Calculation of Strain Information}

FEA using the ANSYS software suite has been conducted to determine the strain distribution in the spiral structure as a result of the imposed loads on the GSS. This can then be used in conjunction with an understanding of the capabilities of the previously developed $3 \mathrm{D}$ printable strain sensors [11,10].

Several modelling decisions were made in the execution of the FEA. A real GSS was approximated by a swept profile for the purpose of this example. The resulting 3D CAD model of a spiral, as shown in Fig. $5 \mathrm{~b}$, was divided into hexahedral mathematical volumes in a process referred to as "meshing". To capture the strain distribution sufficiently, two mesh elements through the thickness of the spiral profile were imposed. The length of the swept profile was divided into 500 mesh elements. The central column, which supports the helical spiral shape in reality, was omitted for simplicity and replaced by a zero-displacement boundary condition in the model. The operational loads on a GSS were represented by a $10 \mathrm{~kg}$ downwards force distributed over the spiral surface. Standard earth gravitational acceleration was enabled in the simulation. Finally, the model was assigned a linear-elastic material with a Young's Modulus of 201.8 MPa, a Poisson's ratio of 0.49 and a yield strength of $73.5 \mathrm{MPa}$.

The resulting elastic strain distribution of the ANSYS simulation is shown in Fig. 7. This result is also exported as a point cloud, then voxelised (averaged and normalised) such that it can be fused with the calculated printability value for each voxel location.

\subsection{Select Sensor Location and Sensor Voxel Sets}

As mentioned earlier, initial sensor locations have been selected by the user based on the domain knowledge and considering various aspects like overlapping sensors to reduce the complexity of having a large number of orientations and locations in a large structure. Using Matlab, the $V_{l o c}$ set has been selected in a rectangular shape (length - 9 voxels, width - 8 voxels), which is similar to the base of the strain gauge. Then, for each $V_{l}$, $V_{s}$ in the shape "U" shape (length - 4 voxels, width - 5 voxels, thickness - 1 voxel) a strain gauge has been selected. Fig. 8 8 shows all $V_{l}$ that are selected and Fig. $8 \mathrm{p}$ shows the bottom-up closer view. In Fig. 8a, some voxels sets are missing at the edge of the spiral, since strain point clouds exported from ANSYS become sparse at the end of the spiral. Thus, there are voxels without any averaged strain values that cause blank spots in $V_{l}$. In these experiments, the locations in $V_{l}$ that contain more than $80 \%$ empty voxels are removed. A plausible alternative solution could be to create a more finely distributed point cloud. In Fig. \&8; sensor-encapsulating areas sometimes have a slightly different number of voxels. This is due to errors that happen when approximating a shape with voxels. This error can be reduced by increasing the granularity of the voxels.

\subsection{Calculation of Percentage of Penalised Voxels}

In this example, placing strain sensors at the outer diameter of the spiral is not ideal since this area is not subjected to a significant amount of strain from the load thus a set of voxels closer to the inner diameter are selected, as shown in Fig. 55. As explained in the Section 2.4.4, the percentages are then calculated for each $V_{l}$. 


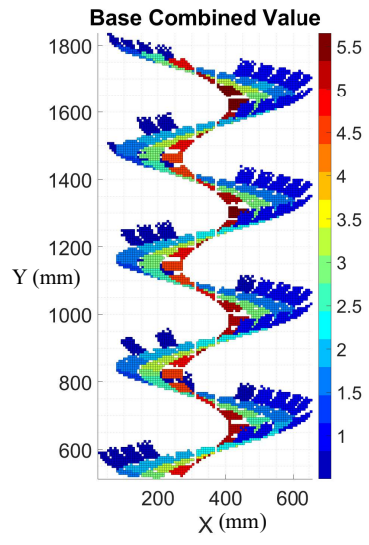

(a)

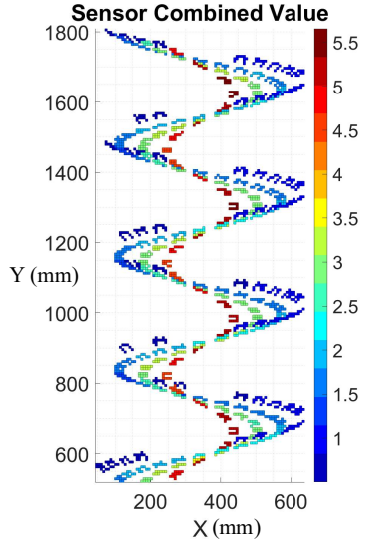

(b)

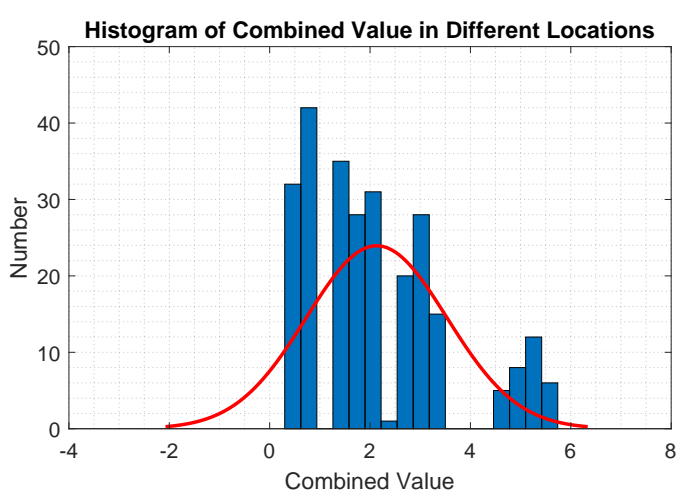

(c)

Fig. 9: Weighted normalised combined objective function values: a) for $V_{l}$; b) for $V_{s}$; c) histogram.

\subsection{Objective Function Evaluation to Select Best Locations}

The weights in the objective functions have been tuned according to the importance of different factors. For the experiments the following values are selected $W_{S}=$ $3, W_{B}=1.5, W_{R}=1$ for the weight of the sensor voxels, weight of the base voxels and weight of the penalised areas, respectively. Since printed traces measure the strain they are subjected to, a higher weight value for $V_{s}$ has been assigned. As such, the weight values are selected to bias towards more traces. After calculating the value for the objective function for each $V_{l}$, each location is ranked and the top ranking locations are se-

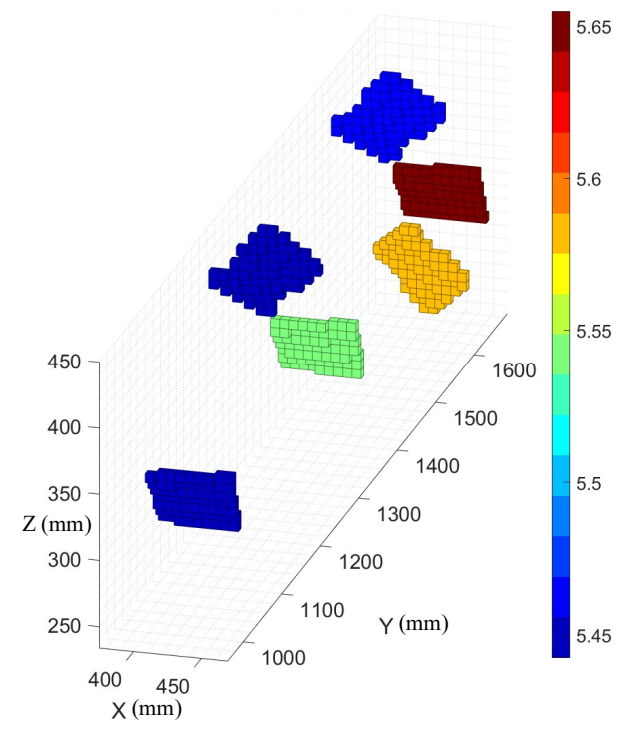

Fig. 10: Six locations with highest objective value (colours represent the weighted unit-less normalised values). lected depending on the number of sensors that need to be placed. Fig. $9 \mathrm{a}$ and b show the calculated combined value from the objective function for $V_{l}$ and $V_{s}$. There are 316 total sensor locations and Fig. 95 shows the distribution histogram for the calculated combined values of the objective function. The mean of the objective function value is 2.13 , and the minimum and maximum values are 0.41 and 5.65, respectively. From Fig. $9 \mathrm{a}$ and $\mathrm{b}$, it is clear that the locations near to the centre is the most suitable because of its higher objective function value. Table 1 shows the six location with the highest values where $W I_{B}=W_{B} \cdot I_{B}, W M_{B}=$ $W_{B} \cdot M_{B}, W I_{S}=W_{S} \cdot I_{S}, W M_{S}=W_{S} \cdot M_{S}, W I_{R}=$ $W_{R} \cdot R_{l}$. This observation aligns with the fact that the highest values of manipulability and strain are also distributed in the same area, as shown in Fig. $5 \mathrm{~d}$ and Fig. 7. Additionally, Table 1 shows a value of zero for $W I_{R}$ and this aligns with the observation that near the central column, there are no voxels where sensor placement is penalised (Fig. 5). A value of zero for $W I_{R}$ indicates that the selected locations are sensible. Fig. 10 shows the six locations with the highest objective values meaning that they are the most suitable locations for sensors, according to the criteria that were selected. To compare different aspects in the objective function, a spider plot has been created with all weight five as-

Table 1: Weighted values of the selected locations

\begin{tabular}{|l|l|l|l|l|l|l|}
\hline Loc. & $W I_{B}$ & $W M_{B}$ & $W I_{S}$ & $W M_{S}$ & $W I_{R}$ & $C_{l}$ \\
\hline 1 & 0.40 & 1.38 & 0.80 & 2.86 & 0 & 5.44 \\
\hline 2 & 0.43 & 1.38 & 0.89 & 2.86 & 0 & 5.55 \\
\hline 3 & 0.45 & 1.38 & 0.97 & 2.86 & 0 & 5.65 \\
\hline 4 & 0.41 & 1.35 & 0.84 & 2.84 & 0 & 5.44 \\
\hline 5 & 0.40 & 1.35 & 0.86 & 2.84 & 0 & 5.45 \\
\hline 6 & 0.46 & 1.35 & 0.93 & 2.85 & 0 & 5.59 \\
\hline
\end{tabular}


pects, as shown in Fig. 11. Since all these locations are not within the area where sensor placements are discouraged, $W I_{R}$ is zero. From the Fig. 11, the change of $W I_{B}$ is $0.06, W M_{B}$ is $0.03, W I_{S}$ is $0.17, W M_{S}$ is 0.02 , $C_{l}$ is 0.21 and therefore, each location is determined to have similar values.

\section{Discussion}

Sensor placement is a critical aspect of structural health monitoring. Optimisation of the sensor placement locations can improve the quality of the data and reduce the instances of economically inefficient false positives 12 , 14. In this application, there are several factors specific to $3 \mathrm{D}$ printed sensor placement in $3 \mathrm{D}$ printed objects that need to be addressed. This paper proposed a way to place $3 \mathrm{D}$ printed sensors into $3 \mathrm{D}$ printed objects by considering the shape, volume and design of the sensor, information gain from the sensors, ability to print the sensors in the given locations, and areas where the sensors should not be placed. To consider the shape and the volume of the sensor, voxelisation of the sensor and the object has been proposed and this methodology has been demonstrated using a "U"-shaped strain sensor. Since measuring strain is the goal of this sensor placement, a FEM simulation has been performed to determine the strain distribution in the GSS. To consider the capabilities of the printer itself, a previously proposed manipulability measure for $3 \mathrm{D}$ printing robots [6] has been incorporated. A simulation environment in Matlab with the actual dimensions of the printer has been developed and used to simulate the printing and generated a distribution of the manipulability as a point cloud. To avoid placing sensors in certain locations, the offending voxels can be penalised. A weighted

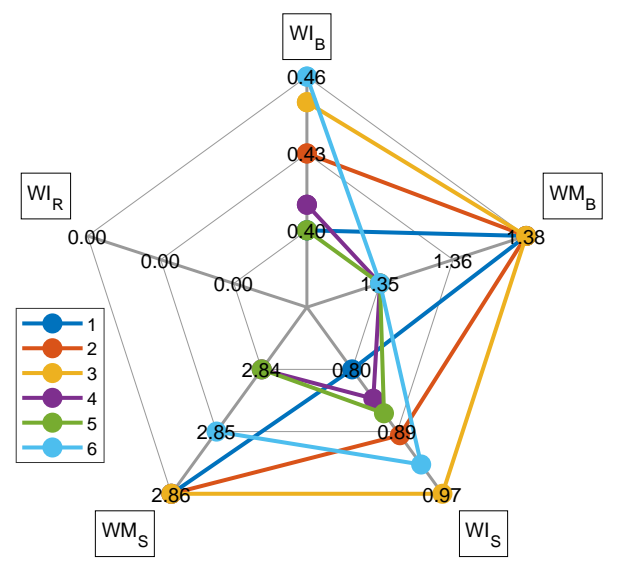

Fig. 11: Spider plot of selected top six positions (weighted normalised values - no unit). objective function has been used to combine all these aspects according to the importance for a given application. In the final step, a set of sensor locations with the highest objective value has been selected. The objective of this experiment is to identify ideal sensor locations that consider all three criteria. The experimental results in Fig. 5 d and Fig. 7 show that the strain and manipulability distribution of the spiral has higher values near the central area of the spiral. Therefore, these locations near the central area of the spiral should be good locations to place sensors. From the experiment results, as shown in Fig. 10 it is clear that the locations suggested by the proposed method align with this observation. Additionally, Table 1 shows that the objective function values for these selected locations and, by comparing these values with the histogram in Fig. 9 it is clear that they reside in the highest values areas compared to all other sensor locations. Therefore, these selected locations are the best locations according to the selected criteria defined by the weighted objective function. The advantage of using this systematic approach, rather than placing sensors using observation, is a broader applicability to more complex 3D printed objects. The number of sensors in this research is selected by the user but can be calculated based on the amount of information extracted from the sensors in relation to the total information available. Initial sensor locations, weights in the objective functions were selected by the user, based on the domain knowledge. These problems are planned to be investigated in future research. This proposed method can be generalised and adapted to place any 3D printed sensor in any larger $3 \mathrm{D}$ printed object since voxelisation and the calculation of manipulability is applicable for any robot arm-based $3 \mathrm{D}$ printing.

\section{Conclusion}

Optimal sensor placement is an important aspect of structural health monitoring. Researchers have proposed various methods to judge candidate sensor locations, like information gain, and different algorithms to identify the optimal placement locations. This paper addressed the optimal 3D printed sensor placement in $3 \mathrm{D}$ printed objects. The proposed method is based on objective functions that consider: the information gain as calculated using FEA; the design of the sensor using weighted voxels; the robots manipulability while printing, or printability; and discouraging sensor placement in certain locations by penalising these voxel sets. The experimental results showed that the presented method can be used to identify locations that incorpo- 
rate and optimise for all these factors when performing $3 \mathrm{D}$ printed sensor placement.

\section{Acknowledgement}

This research is supported by UTS, The Commonwealth of Australia's Department of Industry, Innovation and Science (Innovative Manufacturing CRC Ltd) and

Downer, via its subsidiary Mineral Technologies. Thank you to Rapido, in particular, Hervé Harvard and Michael Behrens for establishing this overall research activity and leading the overall R\&D engineering project. Authors acknowledge that Jordan Henry created the simulation environment of the printer according to the actual dimensions. Thank you to UTS:RI/CAS for providing the required resources to carry out this research.

\section{Declarations}

- Funding: This research is supported by UTS, The Commonwealth of Australia's Department of Industry, Innovation and Science (Innovative Manufacturing CRC Ltd) and Downer, via its subsidiary Mineral Technologies.

- Conflicts of interest/Competing interests: N/A

- Availability of data and material: N/A

- Code availability: N/A

\section{References}

1. IMCRC, "Innovative Manufacturing CRC Annual Highlights 2017-2018," Tech. Rep., 2018.

2. "ISO/ASTM 52900," Tech. Rep., 2015.

3. J. Jiang, X. Xu, Y. Xiong, Y. Tang, G. Dong, and S. Kim, "A novel strategy for multi-part production in additive manufacturing," The International Journal of Advanced Manufacturing Technology, vol. 109, no. 5, pp. 12371248, 2020.

4. K. V. Wong and A. Hernandez, "A Review of Additive Manufacturing," ISRN Mechanical Engineering, vol. 2012, no. 208760, p. 10, 2012.

5. Engineers Australia, "Milestone for mining manufacture with 3D printing," 2019. [Online]. Available: https://portal.engineersaustralia.org. $\mathrm{au} /$ news/milestone-mining-manufacture-3d-printing

6. N. Munasinghe and G. Paul, "Radial slicing for helicalshaped advanced manufacturing applications," The International Journal of Advanced Manufacturing Technology, vol. 112, no. 3-4, pp. 1089-1100, 2020.

7. _ "Path Planning for Robot Based Radial Advanced Manufacturing Using Print Space Sampling," in Int. Conf. on Control, Automation, Robotics and Vision, 2020 .
8. —_ "Integrated 3-D Printable Temperature Sensor for Advanced Manufacturing," in Australasian Conference on Robotics and Automation, 2020.

9. M. I. N. P. Munasinghe, L. Miles, and G. Paul, "DirectWrite Fabrication of Wear Profiling IoT Sensor for 3D Printed Industrial Equipment," in Int. Symposium on Automation and Robotics in Construction, 2019, pp. 862-869.

10. N. Munasinghe, J. Masangkay, and G. Paul, "Temperature Compensated 3D Printed Strain Sensor for Advanced Manufacturing Applications," in IEEE Int. Conf. on Robotics and Automation, 2021.

11. N. Munasinghe, M. Woods, L. Miles, and G. Paul, "3-D Printed Strain Sensor for Structural Health Monitoring," in IEEE Int. Conf. on Cybernetics and Intelligent Systems and the Int. Conf. on Robotics, Automation and Mechatronics, 2019.

12. Y. Tan and L. Zhang, "Computational methodologies for optimal sensor placement in structural health monitoring: A review," Structural Health Monitoring, 2019.

13. H. Sohn, C. R. Farrar, F. Hemez, and J. Czarnecki, "A Review of Structural Health Monitoring Literature," Library.Lanl.Gov, pp. 1-7, 2001.

14. W. Ostachowicz, R. Soman, and P. Malinowski, "Optimization of sensor placement for structural health monitoring: a review," Structural Health Monitoring, vol. 18, no. 3, pp. 963-988, 2019.

15. T. H. Yi and H. N. Li, "Methodology Developments in Sensor Placement for Health Monitoring of Civil Infrastructures," International Journal of Distributed Sensor Networks, vol. 8, 2012.

16. R. F. Guratzsch and S. Mahadevan, "Structural Health Monitoring Sensor Placement Optimization Under Uncertainty," American Institute of Aeronautics and Astronautics Journal, vol. 48, no. 7, pp. 1281-1289, 2010.

17. T.-H. Yi, H.-N. Li, and M. Gu, "A new method for optimal selection of sensor location on a high-rise building using simplified finite element model," Structural Engineering and Mechanics, vol. 37, no. 6, pp. 671-684, 2011.

18. D. Kammer, "Sensor Placement for On-Orbit Modal Identification and Correlation of Large Space Structures," in IEEE American Control Conference, no. 1 1990, pp. 2984-2990.

19. C. Papadimitriou and G. Lombaert, "The effect of prediction error correlation on optimal sensor placement in structural dynamics," Mechanical Systems and Signal Processing, vol. 28, pp. 105-127, 2012.

20. C. Papadimitriou, "Pareto optimal sensor locations for structural identification," Computer Methods in Applied Mechanics and Engineering, vol. 194, no. 12-16, pp. 1655-1673, 2005.

21. M. Meo and G. Zumpano, "On the optimal sensor placement techniques for a bridge structure," Engineering Structures, vol. 27, no. 10, pp. 1488-1497, 2005.

22. D. Wu, E. Coatanea, and G. G. Wang, "Employing Knowledge on Causal Relationship to Assist Multidisciplinary Design Optimization," Journal of Mechanical Design, vol. 141, no. 4, 2019.

23. A. R. M. Rao and G. Anandakumar, "Optimal placement of sensors for structural system identification and health monitoring using a hybrid swarm intelligence technique," Smart materials and Structures, vol. 16, no. 6, p. 2658, 2007.

24. S. Fidanova, P. Marinov, and E. Alba, "Ant algorithm for optimal sensor deployment," in Computational intelligence. Springer, 2012, pp. 21-29. 
25. L. Fuqing and Z. Lingmi, "Successive method for optimal placement of actuators and sensors," Journal of Astronautics, vol. 3 , no. 21 , pp. 64-69, 2000.

26. M. Behzadian, R. B. Kazemzadeh, A. Albadvi, and M. Aghdasi, "PROMETHEE: A comprehensive literature review on methodologies and applications," European Journal of Operational Research, vol. 200, no. 1, pp. 198-215, 2010.

27. Addinsoft, "Electre Methods," 2021. [Online]. Available: https://www.xlstat.com/en/solutions/features/ multicriteria-decision-aid-electre-methods

28. H. Hwang, J. H. Bae, and B. C. Min, "Design guidelines for sensor locations on 3D printed prosthetic hands," in IEEE Int. Conf. on Robotic Computing, 2017, pp. 412417.

29. A. Makwe and P. Kanungo, "Scheduling in cloud computing environment using analytic hierarchy process model," in Int. Conf. on Computer, Communication and Control, 2015 , pp. 1-4.

30. Y. Bougdid and Z. Sekkat, "Voxels Optimization in 3D Laser Nanoprinting," Scientific Reports, vol. 10, no. 1, p. 10409, 2020

31. J. E. Lavín-Delgado, J. E. Solís-Pérez, J. F. GómezAguilar, and R. F. Escobar-Jiménez, "A New FractionalOrder Mask for Image Edge Detection Based on Caputo-Fabrizio Fractional-Order Derivative Without Singular Kernel," Circuits, Systems, and Signal Processing, vol. 39, no. 3, pp. 1419-1448, 2020.

32. K. B. Rahmat, D. Dharma, and A. Manaf, "Rendering Speed Improvement for Particle Based Fluid Simulation on GVDB Voxels using Whitted Raytracing," in Int. Conf. on Data and Software Engineering, 2019, pp. $1-6$.

33. W. Wijaya, M. Ali, R. Umer, K. Khan, P. Kelly, and S. Bickerton, "An automatic methodology to CT-scans of $2 \mathrm{D}$ woven textile fabrics to structured finite element and voxel meshes," Composites Part A: Applied Science and Manufacturing, vol. 125, p. 105561, 2019.

34. G. Paul, L. Liu, and D. Liu, "A novel approach to steel rivet detection in poorly illuminated steel structural environments," in Int. Conf. on Control, Automation, Robotics and Vision, 2016, pp. 1-7.

35. T. I. Zohdi, "Rapid Voxel-Based Digital-Computation for Complex Microstructured Media," Archives of Computational Methods in Engineering, vol. 26, no. 5, pp. 13791394, 2019.

36. J. H. Jung, Y. Shin, and Y. Kwon, "Extension of convolutional neural network with general image processing kernels," in IEEE Region 10 Conference, 2018, pp. 14361439.

37. P. Corke, Robotics Vision and Control, 2nd ed. Springer, 2015 , vol. 75 , no. $1-2$.

38. T. Yoshikawa, "Manipulability and redundancy control of robotic mechanisms," in IEEE Int. Conf. on Robotics and Automation, vol. 2, 1985, pp. 1004-1009.

39. P. Corke, "Robotics Toolbox." [Online]. Available: https://petercorke.com/toolboxes/robotics-toolbox/
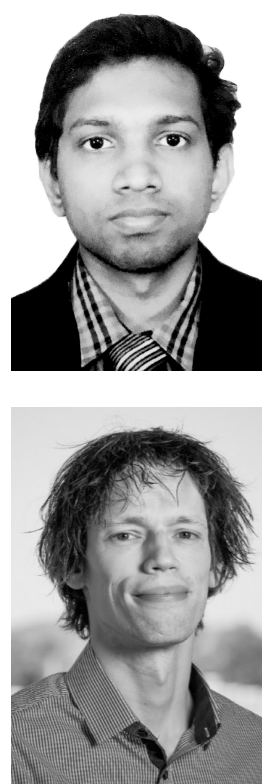

Nuwan Munasinghe received a B.Sc. Eng. (Hons.) degree in Computer Science and Engineering from University of Moratuwa, Sri Lanka, in 2016. He is currently pursuing a $\mathrm{PhD}$ in engineering at the University of Technology Sydney (UTS), Sydney, Australia.

Thomas Romeijn holds a Master's degree in Mechanical Engineering through studies at Dutch, German and Australian universities. $\mathrm{He}$ is a Chartered Professional Engineer and his speciality is the Finite Element Analysis (FEA) method which he has successfully applied for over 16 years in the fields of Stress Analysis, Fluid Flow, Magnetostatics and dynamics, and Electrostatics and dynamics. Thomas is currently undertaking an industry-funded $\mathrm{PhD}$ project at the University of Technology Sydney.

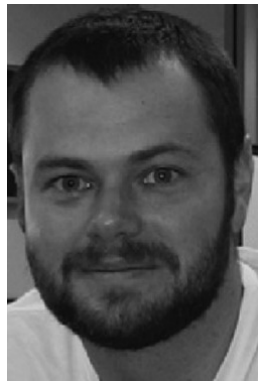

Gavin Paul graduated with a B.Eng. in Computer Systems Engineering at the University of Technology, Sydney (UTS). In 2010 he received his $\mathrm{PhD}$ in Engineering (Robotics) at UTS. 